\title{
Electron cloud detection and characterization in the CERN Proton Synchrotron
}

\author{
Edgar Mahner, Tom Kroyer, and Fritz Caspers \\ CERN, 1211 Geneva 23, Switzerland \\ (Received 2 April 2008; published 16 September 2008)
}

\begin{abstract}
We describe a dedicated electron cloud experiment which was installed in the CERN Proton Synchrotron in 2007. The setup comprises shielded button-type pickups, a fast vacuum logging, a dipole magnet, and a stripline electrode to experimentally verify the beneficial effect of electron cloud clearing. The electron cloud effect was observed within the last milliseconds before ejection of the nominal LHC proton beam consisting of 72 bunches with $25 \mathrm{~ns}$ spacing. Measurements of electron flux at the wall and vacuum pressure are presented for a set of magnetic fields and bias voltages on the clearing electrode, showing that efficient electron cloud suppression can be obtained for appropriate clearing voltages but revealing an unexpectedly complex dependence on magnetic field and voltage.
\end{abstract}

DOI: 10.1103/PhysRevSTAB.11.094401

PACS numbers: 29.27.Bd, 29.20.dk, 79.20.Hx

\section{INTRODUCTION}

Since its first observation at the Budker Institute of Nuclear Physics Proton Storage Ring, the electron cloud (EC) effect has been discovered at many high intensity particle accelerators, including the CERN Intersecting Storage Rings, the Stanford Linear Accelerator Center PEP-II, the CERN Super Proton Synchrotron (SPS), and the KEK B Factory [1-5]. In the CERN Proton Synchrotron (PS) EC effects have been observed in 2000 with the first production of high intensity LHC beam [6]. The indications were a baseline drift in electrostatic beam diagnostics and single bunch instabilities. For the current production scheme of the nominal LHC beam no electron cloud related instabilities are observed, but it is not excluded that such instabilities may occur for higher proton intensities [7]. For this reason it was desirable to have a dedicated EC diagnostics in the PS, which is the subject of this paper.

In the past years in many laboratories large efforts were put into understanding and characterizing the EC by machine studies, numerical simulations, and by dedicated diagnostics. The goal is to mitigate or suppress the EC effect. By tuning the beam parameters the EC can be reduced. However, this method has the drawback that the accelerator performance may be negatively affected. In some cases machine operation in presence of an EC can be handled by damping the induced instabilities.

There are two approaches for suppressing the EC effect: the first is to modify the chamber surface properties such that the secondary emission yield (SEY) is reduced, if possible below unity. This can be at least partially achieved by conditioning the vacuum chamber surface by exposing it to a deliberately generated electron cloud over an extended period, which is often called beam scrubbing [8]. Surface coatings, e.g., nonevaporable getter (NEG) or TiN, directly reduce the SEY and, in the case of activated NEG, the vacuum pressure is improved as well $[9,10]$. SEY reduction by an artificial surface roughness, both in the micrometer or millimeter range, is also being researched $[11,12]$. The second approach consists in changing the dynamics of the EC by externally applied electric, magnetic, or electromagnetic fields. Solenoids have been shown to confine the electrons close to the chamber wall and eliminate the EC in straight sections of several machines $[13,14]$, but they cannot be applied in bending sections. Clearing electrodes allow a static electric field to be applied, which changes the trajectories of the electrons and may disturb the EC buildup. Electromagnetic fields were also proposed for electron cloud clearing [15].

Clearing electrodes have been successfully used in several machines for electron or ion clearing $[14,16,17]$. However, most of the experience is limited to short button-type electrodes. Experiments with longer electrodes are reported in $[16,18,19]$. In order to cover longer sections of an accelerator, many button-type electrodes or a long stripline electrode are needed. As the electrode length increases, issues related to beam coupling impedance and mechanical stability get more stringent. A potential solution with rather low impedance and good mechanical properties consists of resistive layers deposited onto a ceramic or an enamel strip inside the beam pipe [20]. Numerical simulations for different machines showed that clearing electrodes can have a very beneficial effect [14,20-22].

\section{EXPERIMENTAL SETUP}

At the CERN Proton Synchrotron a new electron cloud experiment has been installed in March 2007. The experimental setup was designed, fabricated, and mounted in straight section (SS) 98 during the accelerator shutdown 2006/2007.

The experiment consists of an elliptical 316 LN (low carbon with nitrogen) stainless steel vacuum chamber with $147 \mathrm{~mm}$ horizontal and $72 \mathrm{~mm}$ vertical aperture and a 


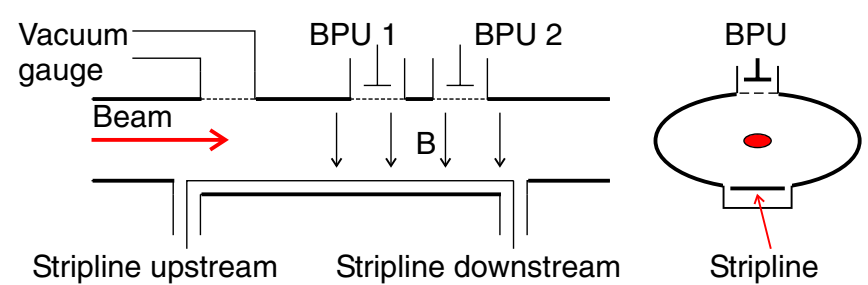

FIG. 1. (Color) Schematic view of the PS electron cloud experiment, comprising a shielded Penning gauge, two shielded button pickups (BPU), a stripline clearing electrode, and a dipole magnet (not sketched). Left: Longitudinal cut, right: transverse cut at the position of a BPU.

length of $1050 \mathrm{~mm}$. Two identical $30 \mathrm{~mm}$ diameter button pickups and a Penning gauge are installed on the upper part of the vacuum chamber. The pickups are both shielded from the main chamber with $0.7 \mathrm{~mm}$ thick perforated stainless steel sheets, which provide a transparency of $10 \%$ for pickup \#1. As a different approach, pickup \#2 is shielded with two higher transparency $(37 \%, 23 \%)$ grids. The Penning gauge is shielded with a $37 \%$ transparency grid and installed behind a $90^{\circ}$ elbow in order to protect it against electrons. All shielding grids are electrically grounded to the vacuum chamber. On the bottom side of the vacuum chamber, a $400 \mathrm{~mm}$ long, $46 \mathrm{~mm}$ wide, and $2 \mathrm{~mm}$ thick $316 \mathrm{LN}$ stainless steel clearing electrode is installed inside a small antechamber in order to avoid any aperture reduction for the beam. The installation is depicted schematically in Fig. 1.

The stripline-type electrode is mounted on and supported by two standard high-voltage feedthroughs. The electrode geometry was optimized for its function as part of an electron cloud diagnostics equipment. The gap between the electrode and the adjacent beam pipe was made small in order not to lose too many electrons in the gap, which would reduce the EC by geometrical means. The line impedance to ground of the electrode is about
$30 \Omega$, which means that the mismatch to the connected $50 \Omega$ coaxial cable is acceptable. As for every new installation, the beam coupling impedance of the chamber had to be checked. Since the vacuum diagnostics port and the button pickups are behind shielding grids, only the stripline electrode has a significant impact on the impedance. By numerical simulations, using CST MICROWAVE STUDIO ${ }^{\text {, }}$, the longitudinal and transverse impedance were found to be of the order of $0.1 \%$ of the present PS impedance and thus negligible.

Since the experiment is mounted in a field-free region in the PS, the central part of the electron cloud detector is surrounded by a small $\mathrm{C}$-shaped dipole magnet that can produce a homogenous magnetic field of up to $100 \mathrm{G}$ [23]. A picture of the experimental area in the PS is shown in Fig. 2.

The signals from the button pickups and from the stripline electrode were transmitted to a local control room using about $44 \mathrm{~m}$ long $50 \Omega$ coaxial low-loss cables. In addition to being a clearing electrode, the stripline was used as a pickup to get a wideband beam signal. A custombuilt frequency separating filter was used to observe the beam signals with the possibility of applying a bias voltage up to $\pm 1 \mathrm{kV}$ on the electrode.

A similar bias network was implemented for the button pickups with a maximum bias voltage of $\pm 500 \mathrm{~V}$. Lownoise preamplifiers were used for observing the signal on a sampling scope. The $3 \mathrm{~dB}$ bandwidth of the entire signal transmission and treatment chain was about 0.045 to $350 \mathrm{MHz}$, which yields a rise time of about $0.94 \mathrm{~ns}$.

\section{RESULTS}

First, the rf gymnastics in the PS should be outlined, since these determine the bunch length, which is a crucial factor for the EC buildup. A more detailed description of the rf manipulations can be found in [24]. For the production of the nominal LHC beam, six bunches are injected
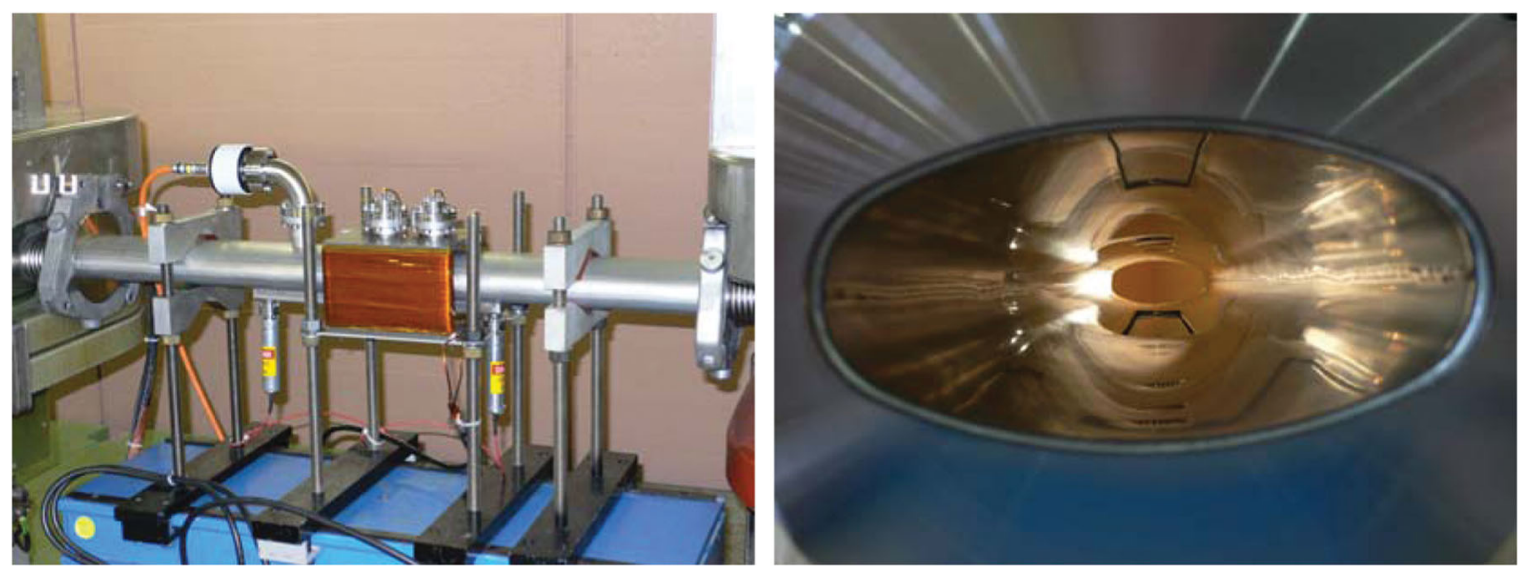

FIG. 2. (Color) Left: Picture of the new electron cloud experiment installed in PS straight section 98, containing two shielded button pickups, a shielded Penning gauge, a clearing electrode, and a small dipole magnet. Right: Downstream view into the vacuum chamber showing the stripline electrode at the bottom. 
TABLE I. Summary of the bunch length evolution during the last milliseconds in the PS cycle.

\begin{tabular}{lcccc}
\hline \hline Operation & Time before ejection & Number of bunches & Bunch spacing & Bunch length $(4 \sigma)$ \\
\hline Second bunch splitting & $57 \mathrm{~ms}$ & 36 & $50 \mathrm{~ns}$ & \\
Third bunch splitting & 27 to $5 \mathrm{~ms}$ & 72 & $25 \mathrm{~ns}$ & $14 \mathrm{~ns}$ \\
Adiabatic bunch compression & 5 to $0.3 \mathrm{~ms}$ & 72 & $25 \mathrm{~ns}$ & $11 \mathrm{~ns}$ \\
Bunch rotation & 0.3 to $0.0 \mathrm{~ms}$ & 72 & $25 \mathrm{~ns}$ & $4 \mathrm{~ns}$ \\
\hline \hline
\end{tabular}

TABLE II. The main machine and beam parameters for the PS electron cloud experiment.

\begin{tabular}{lc}
\hline \hline PS circumference & $628 \mathrm{~m}$ \\
Proton energy & $25 \mathrm{GeV}$ \\
Revolution time & $2.1 \mu \mathrm{s}$ \\
Number of bunches & 72 \\
Bunch spacing & $25 \mathrm{~ns}$ \\
Bunch length & $4 \mathrm{~ns}$ \\
Bunch population & $1.1 \times 10^{11} \mathrm{protons} / \mathrm{bunch}$ \\
Bunch emittance & $0.35 \mathrm{eVs}$ \\
Vacuum chamber aperture (horizontal) & $147 \mathrm{~mm}$ \\
Vacuum chamber aperture (vertical) & $72 \mathrm{~mm}$ \\
\hline \hline
\end{tabular}

into the PS. After a triple splitting the beam is accelerated to the top energy of $25 \mathrm{GeV}$, where the second and third bunch splittings are performed to yield 72 bunches with a spacing of $25 \mathrm{~ns}$. Before ejection the bunches are first shortened adiabatically over $5 \mathrm{~ms}$ and then by a fast phase space rotation within $300 \mu \mathrm{s}$. The evolution of the bunch length is summarized in Table I. The EC effect is expected, in particular, right before PS ejection, when bunches are shortened for injection into the SPS. Table II summarizes the machine conditions during the electron cloud experiments.

\section{A. Pressure rise}

The commissioning of the new experimental setup and first data taking took place before and during the SPS scrubbing run in June 2007. As a first indication, pressure rises were recorded with the Penning gauge in straight section 98 during the presence of the nominal LHC beam in the PS machine. A typical result is shown in Fig. 3, which displays the pressure evolution with time for a PS supercycle that contained sequences of four successive nominal 72-bunch LHC beams every $3.6 \mathrm{~s}$.

A systematic and repetitive pressure rise of $\Delta p \approx 3 \times$ $10^{-8}$ mbar was measured for each of the four consecutive LHC beams. The pressure maxima appear when the proton beam is extracted from the PS. Each of these larger pressure peaks is accompanied by a smaller peak which starts about $800 \mathrm{~ms}$ before extraction [see Fig. 3 (right)]. This time corresponds very well with the transition crossing of the protons in the PS, which is exactly $830 \mathrm{~ms}$ before beam extraction. Pressure changes were not visible with other non-LHC-type beams accelerated within the same
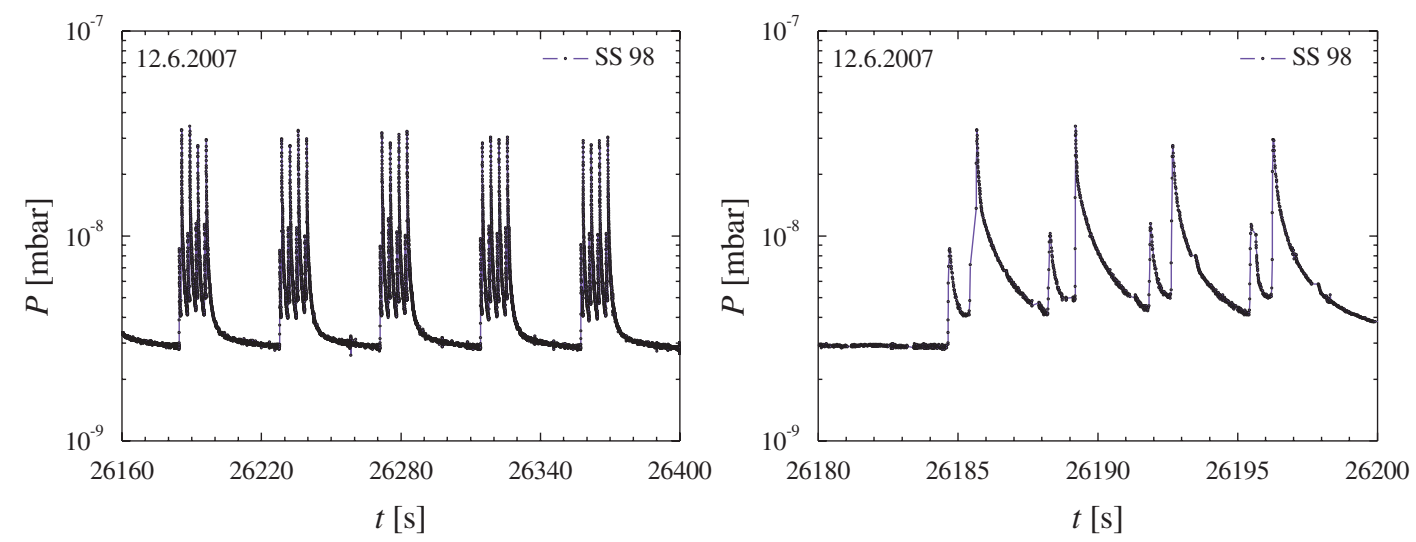

FIG. 3. (Color) Pressure rises in PS straight section 98 measured on 12/6/2007 at 21:38 during a PS supercycle that contained four nominal LHC beams. Right: Zoom into the pressure rise caused by $4 \times 72$ bunches. The distance between the main peaks is $3.6 \mathrm{~s}$, the smaller peaks appear about $0.8 \mathrm{~s}$ before. 
supercycle in the PS [see Fig. 3 (left)]. We also did not observe a pressure-run-away since the duty cycle of the LHC beam in the PS is rather low and because there is enough pumping speed available close to the experiment in SS98. Therefore, the vacuum recovers rather quickly before $3.6 \mathrm{~s}$ later the next LHC beam is injected, accelerated, and extracted.

A much higher pressure rise up to about $10^{-6}$ mbar was measured in SS98 during the PS setting up for the SPS scrubbing run. This effect was probably related to increased proton losses during that period.

\section{B. Button pickup signals}

The presence of an electron cloud was also immediately seen with both button pickups. The results obtained for the last four turns before extraction of the nominal LHC beam from the PS are displayed in Fig. 4. The beam-induced signal observed on the clearing electrode, which acts essentially as a stripline beam position monitor, was used for synchronization and to monitor the basic beam properties. On both button pickups a clear electron signal was seen when the LHC beam passed. The time $t=0 \mathrm{~s}$ was set to the position of the last bunch at the last turn before ejection. It can be seen that at the last turn both the beam signal on the stripline as well as the electron cloud signal are smaller than during the previous turns. This is due to the orbit offset right before ejection, which moves the beam away from the stripline electrode and changes the EC dynamics.

Systematic measurements were performed by changing the bias voltage on the pickups between $+500 \mathrm{~V}$ and $-500 \mathrm{~V}$, showing that for negative voltages the arriving electrons can be almost completely suppressed at the button pickups, while at large positive voltages the electron current tends to "saturate," indicating that most of the electrons passing across the grid are captured on the buttons. For practical reasons a bias voltage of $+60 \mathrm{~V}$ has been chosen for all further measurements.

Because of the high bandwidth $(350 \mathrm{MHz})$ of the system every $4 \mathrm{~ns}(4 \sigma)$ long bunch can be resolved on the stripline electrode and on the button pickups. Measurements during the second last turn, showing the start of the electron cloud

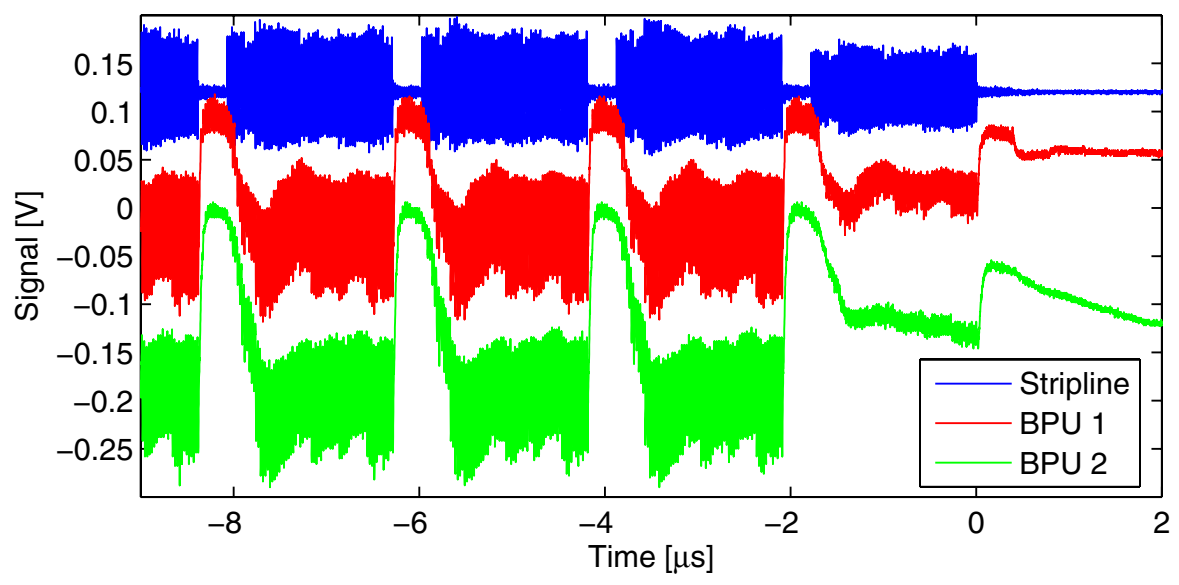

FIG. 4. (Color) Measured signals of the stripline electrode and the button pickups \#1 and \#2 during the passage of the nominal LHC beam. The last 4 turns before extraction from the PS are shown, the gap between successive recurrences of the same group of 72 bunches is $320 \mathrm{~ns}$. The traces have been stacked vertically for clarity.
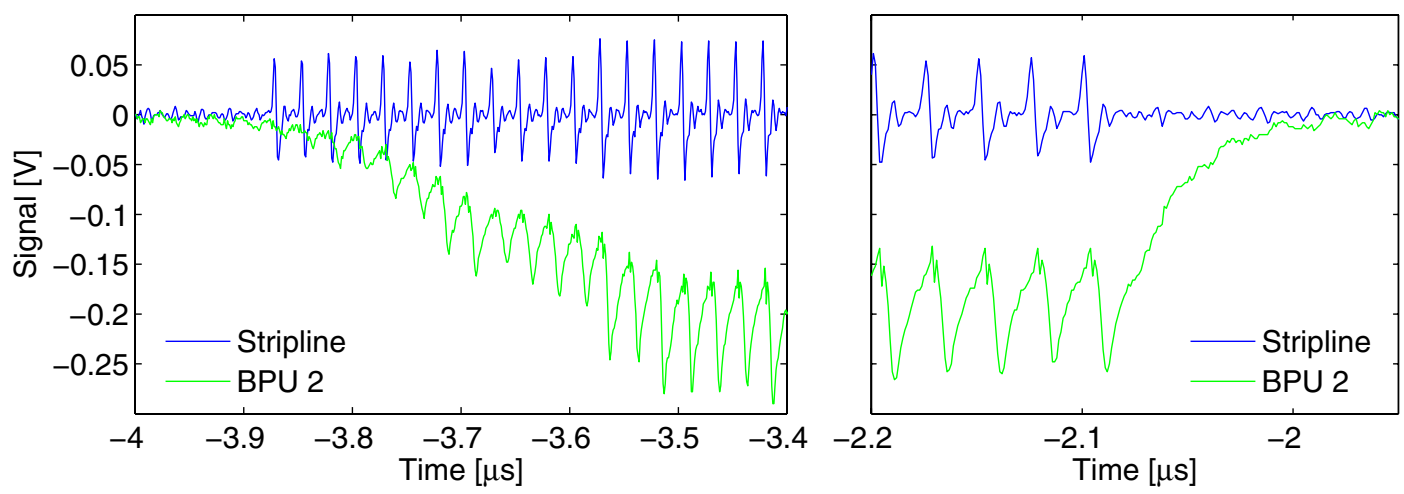

FIG. 5. (Color) Bunch-to-bunch electron cloud buildup (left) and decay (right) measured with button pickup \#2 during the second last turn of the LHC beam. The 25 ns gap between successive bunches can be clearly resolved with the stripline electrode. 


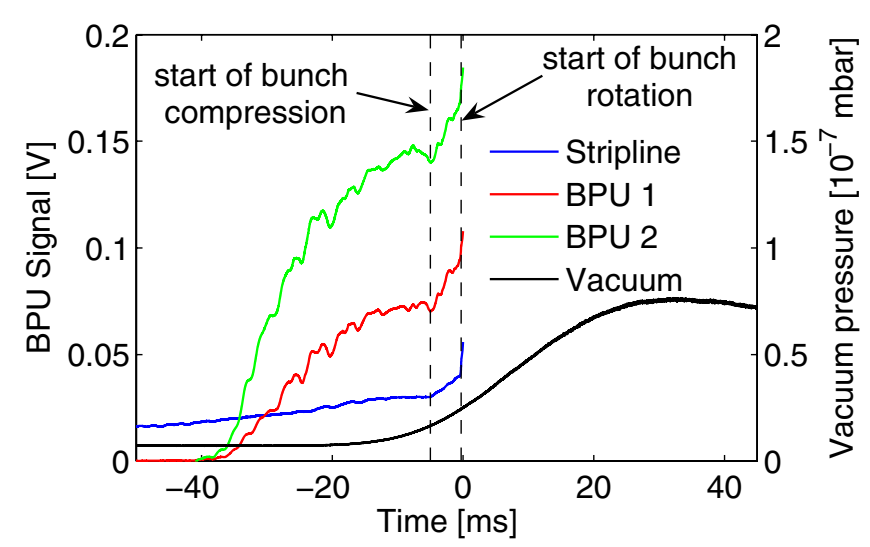

FIG. 6. (Color) The pickup signals (BPU 1 and BPU2) and the stripline peak signal (in arbitrary units) extracted from the raw data together with the vacuum pressure for the nominal LHC beam with 72 bunches. The EC becomes visible on the pickups about $40 \mathrm{~ms}$ before ejection, the vacuum gauge follows with a delay of about $30 \mathrm{~ms}$.

buildup with the first of the 72 bunches and decrease behind the last bunches, are displayed in Fig. 5.

In order to determine the detection limit of the experiment, the noise level of the pickups was measured. For that purpose the electrons collected by the pickups were suppressed as much as possible by applying a high negative voltage of $-500 \mathrm{~V}$. The remaining signal can be classified into two categories: First, a slow ripple with a frequency of $\sim 2 \mathrm{MHz}$ and a peak-to-peak voltage of $\sim 5 \mathrm{mV}$ probably picked up by the long cables from the PS machine to the local control room; second, rather sharp peaks following very closely the LHC beam signal which is probably related to a direct coupling of the proton beam across the shielding grids of the pickups resulting in a peak-to-peak voltage of $\sim 5 \mathrm{mV}$ for pickup \#1 and $\sim 10 \mathrm{mV}$ for pickup \#2.

The measured pickup signals can be used to estimate the electron line density for our experiment. The current on one button $I_{\mathrm{pu}}$ and the measured voltage $U_{\mathrm{pu}}$ are given by

$$
I_{\mathrm{pu}}=\lambda e f_{b} \frac{A_{\mathrm{pu}}}{A_{\mathrm{ch}}} t \quad U_{\mathrm{pu}}=I_{\mathrm{pu}} \cdot Z_{0} \cdot A \cdot G,
$$

where $\lambda$ is the electron line density, $e$ is the electron charge, $f_{b}$ is the bunch frequency, $A_{\mathrm{pu}}$ is the surface of the pickup button, $A_{\mathrm{ch}}$ is the inner surface of the vacuum chamber per meter, $t$ is the button transparency, $Z_{0}$ is the system impedance, $A$ is the cumulated attenuation of cables and filters, and $G$ is the amplifier gain of the measurement circuit. It is assumed that the current of the electrons impinging on the wall is homogeneous in the center of the beam pipe and that no electrons are lost on the side walls of the grid as will be the case for a thin grid. For an EC appearing in stripes, as is usually found with magnetic fields, deviations from the above formula are expected, which cannot be avoided with button-type pickups.

Combining all the factors the line density can be estimated from

$$
\frac{\lambda}{\mathrm{e}^{-} / \mathrm{m}}=\frac{U_{\mathrm{pu}}}{\mathrm{mV}} \cdot 2.3 \times 10^{8} .
$$

Therefore, with a measured button pickup voltage of about $250 \mathrm{mV}$ (see Fig. 4, BPU 1), we obtain an electron line density of $\lambda \approx 5.8 \times 10^{10} \mathrm{e}^{-} / \mathrm{m}$.

For further evaluations the data were analyzed turn-byturn using a MATLAB ${ }^{\circledR}$ script. For each machine turn the

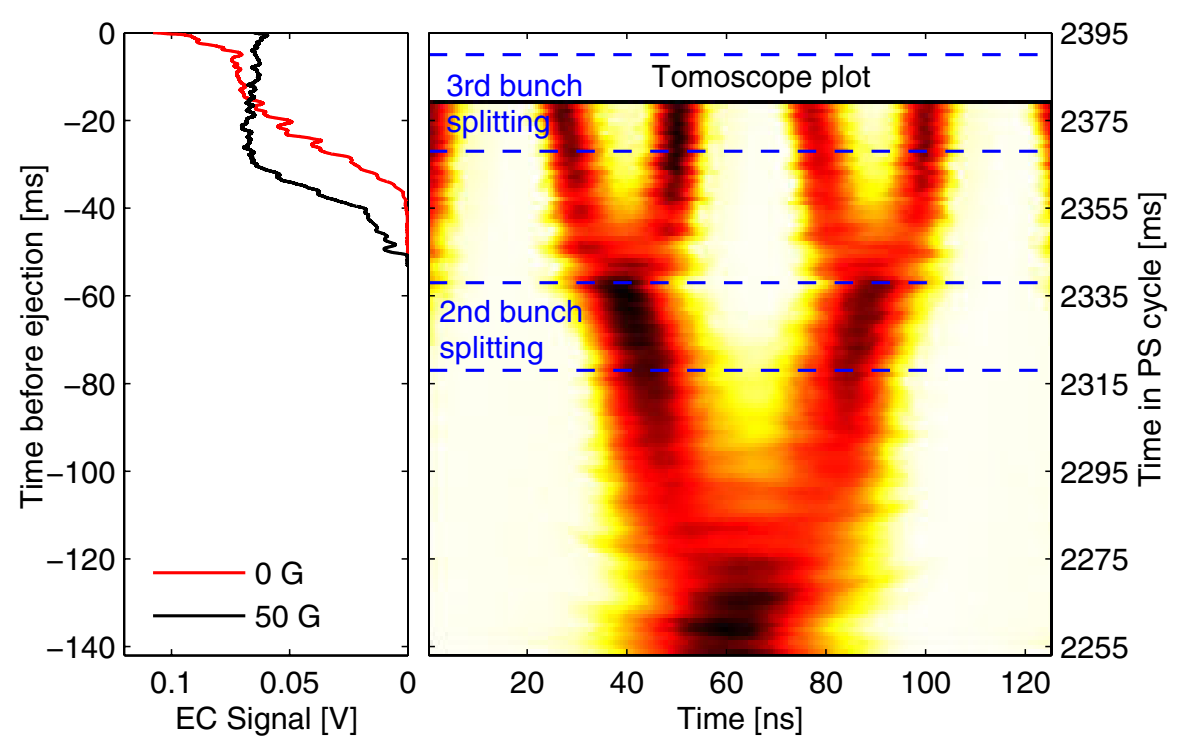

FIG. 7. (Color) The EC signal (left) compared with the bunch length during the rf gymnastics represented in a tomoscope plot (right, courtesy of Steven Hancock.). The EC buildup starts 40 to $50 \mathrm{~ms}$ before ejection, right after the second bunch splitting. 


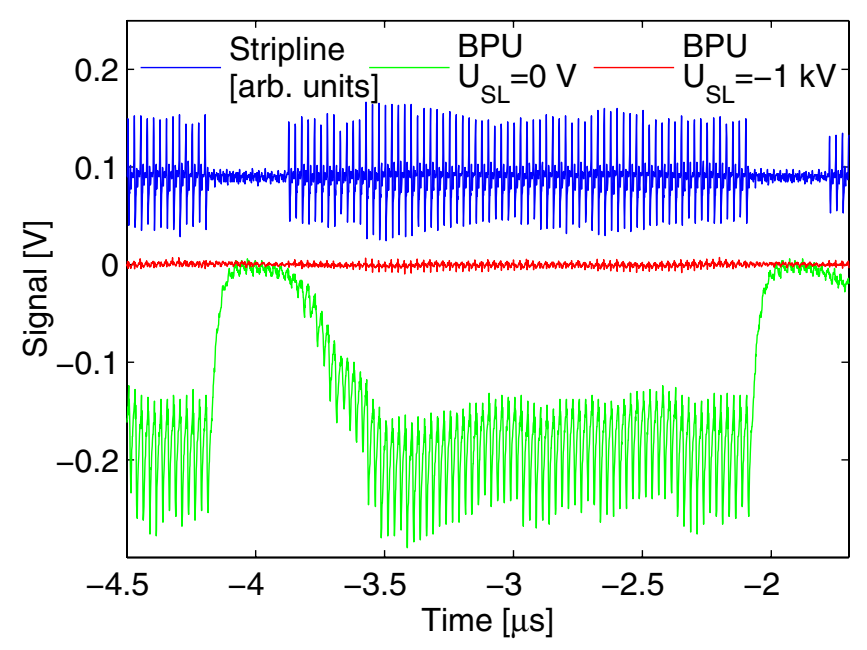

FIG. 8. (Color) The electron cloud was suppressed below the detection limit for large enough clearing voltages, e.g. $U_{\mathrm{SL}}=$ $-1 \mathrm{kV}$, data from BPU 2 are plotted.

peak beam signal on the stripline electrode, the maximum EC signal on the pickups, the EC buildup time, and the EC decay time were determined. The stripline peak signal and the EC signal on the pickups were averaged over the last 12 bunches of each turn, which gives a considerable increase in sensitivity.

Figure 6 shows the pickup signals together with the beam signal on the stripline and the vacuum pressure. Because of the bunch splitting (27 to $5 \mathrm{~ms}$ before ejection), the maximum beam signal increases first slowly, then faster during the last $5 \mathrm{~ms}$ (bunch compression), and still faster during the bunch rotation (last $300 \mu \mathrm{s}$ ). The EC effect kicks off about $40.7 \mathrm{~ms}$ before ejection and grows

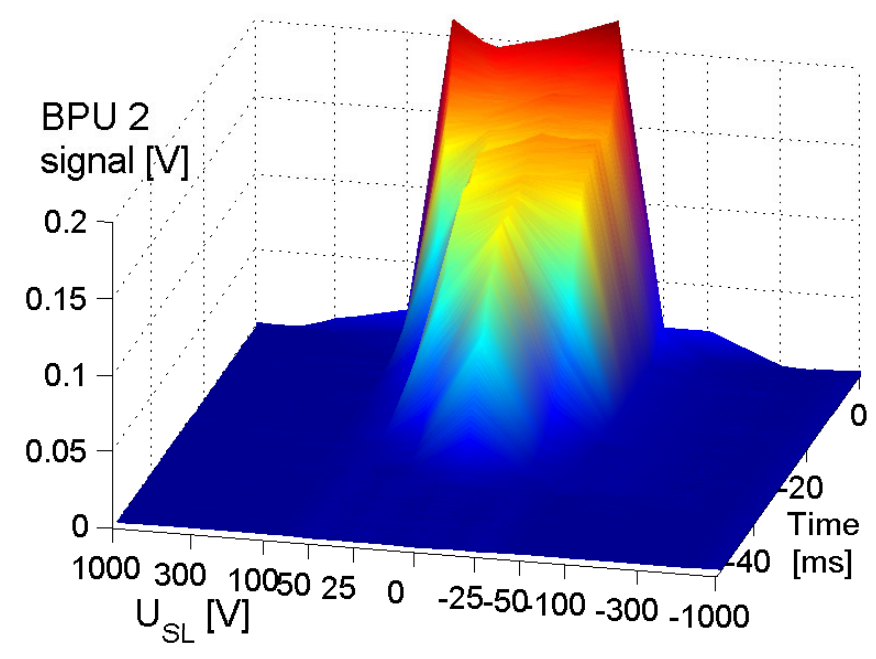

FIG. 9. (Color) Electron cloud button pickup \#2 signal measured in SS98 for the nominal LHC beam. The color-coded signal height varies as a function of applied voltage on the clearing electrode and with time, no magnetic field was applied. The last $50 \mathrm{~ms}$ before ejection are shown. more intense during the last bunch splitting. During bunch compression and rotation, the EC gets still more intense.

The EC effect is also clearly visible on the vacuum diagnostics, which follows the pickup signals with a delay of about $30 \mathrm{~ms}$. The observed vacuum rise times are of the order of $25 \mathrm{~ms}$, which could still be measured with the Penning gauge (10 to $90 \%$ rise time of $10 \mathrm{~ms}$ ).

Some systematic measurements were performed to determine the exact time when the electron cloud buildup starts to be detectable in our experiment. In Fig. 7 the EC signal on pickup \#1 is compared to a tomoscope plot of the longitudinal bunch profile. The EC is observed well before the end of the last bunch splitting (27 to $5 \mathrm{~ms}$ before ejection).

\section{Effect of the clearing electrode}

In order to study the possibility of influencing or even suppressing the electron cloud locally, negative and positive voltages were applied on the stainless steel clearing electrode which is located opposite to the button pickups. For large enough positive and negative clearing voltages $\left|U_{\mathrm{SL}}\right|>300 \mathrm{~V}$, the EC signals decreased below our detection limit. The case of $U_{\mathrm{SL}}=-1 \mathrm{kV}$ is depicted in Fig. 8 .

The pickup signals have been measured for clearing voltages ranging from $-1000 \mathrm{~V}$ to $+1000 \mathrm{~V}$. Figure 9 shows the EC signal on pickup \#2 averaged over the last 12 of 72 bunches in the PS machine as a function of clearing voltage. The data cover the last $50 \mathrm{~ms}$ before the proton beam is ejected from the PS. Without magnetic field the EC is suppressed almost equally well for both polarity clearing voltages. When the clearing voltage is not large enough such as to completely suppress the EC at least the buildup sets on later.

\section{Effect of a dipole field}

The C-shaped dipole magnet (see Fig. 2) has been used to study the electron cloud effect for magnetic fields up to $B=70 \mathrm{G}$. In addition, for each magnetic field studied, the clearing voltage $U_{\mathrm{SL}}$ has been varied between $-1000 \mathrm{~V}$ and $+1000 \mathrm{~V}$ resulting in 65 individual measurements. The EC signal is plotted as a function of magnetic field and clearing voltage in the frames of Video 1 for crucial points in the PS cycle, Video 1 shows the time evolution of the pickup signal. The amplitude of the pickup \#1 signal is color coded. The signals were averaged over the last 12 bunches of 72. It can be observed from the first plot $(t=$ $-45 \mathrm{~ms}$ ) that with a magnetic field the EC kicks off earlier (see also Fig. 7). Later in the PS cycle, islands with large $\mathrm{EC}$ form in the parameter space. In particular for small negative clearing voltages and with magnetic field the EC may even be enhanced. However, for sufficiently large positive or negative clearing voltages (close to $1 \mathrm{kV}$ ) EC clearing could always be achieved at the location of the pickups. 

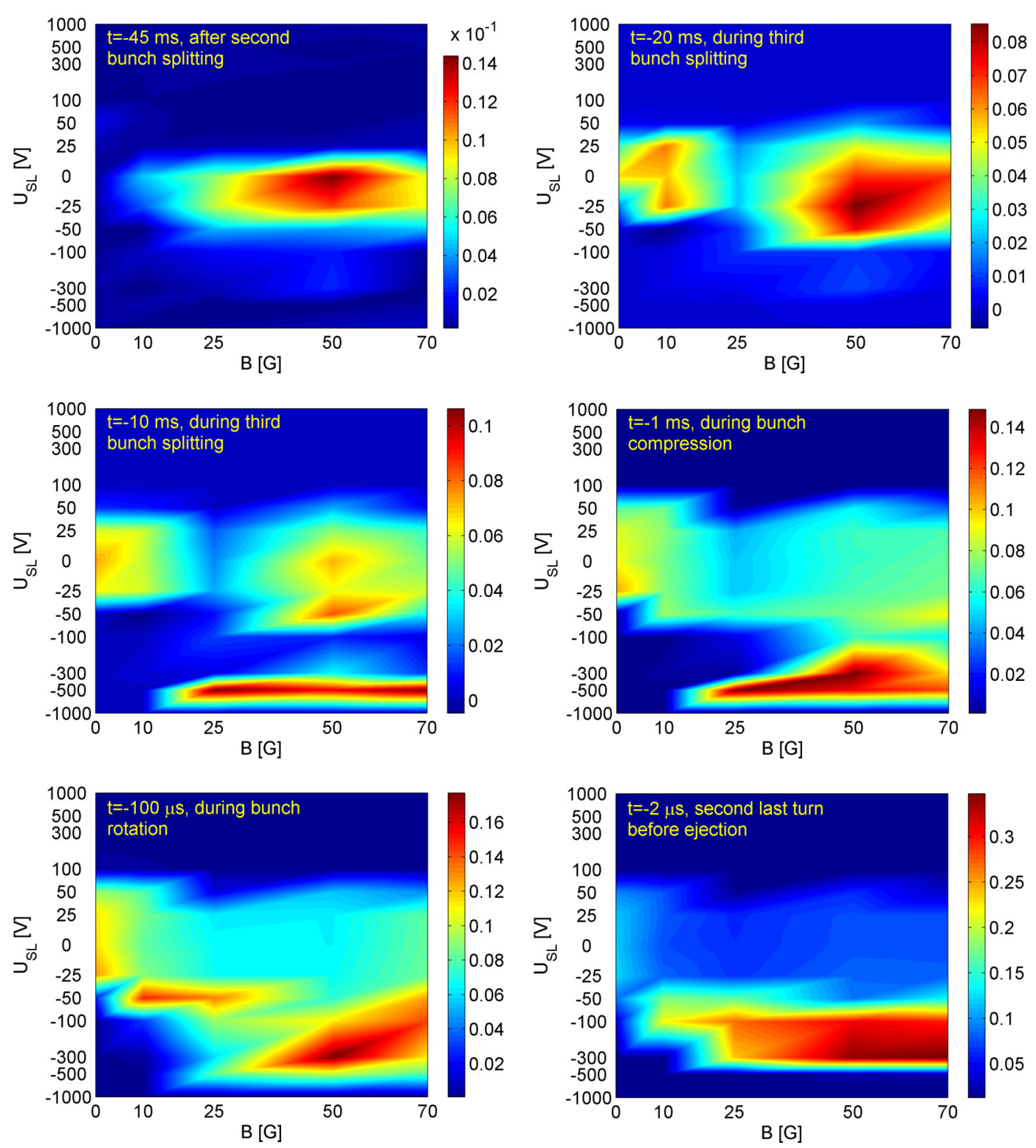

VIDEO 1. Electron cloud signal on pickup \#1 measured for various clearing electrode voltages $\left(-1 \mathrm{kV} \leq U_{\mathrm{SL}} \leq+1 \mathrm{kV}\right)$ and magnetic dipole fields $(0 \mathrm{G} \leq B \leq 70 \mathrm{G})$. Measurements were taken continuously during the last $50 \mathrm{~ms}$ before beam extraction at $t=0 \mathrm{~ms}$. This video shows the time evolution of the pickup signal.

\section{E. Vacuum pressure evolution with time}

The vacuum pressure was logged for the whole range of magnetic fields and clearing voltages examined. A fast vacuum pressure rise was observed which is triggered by the EC. Averaged over the vacuum diagnostics rise time of $10 \mathrm{~ms}$ the EC effect is stronger with magnetic field than without. In the frames of Video 2 measurements taken at 0 and $50 \mathrm{G}$ are compared; the time evolution of the vacuum pressure is shown in Video 2. The vacuum pressure rise mirrored the observations on the pickups: with magnetic field the pressure bump starts earlier and is stronger than without. However, even with large clearing voltages on the stripline, a delayed pressure rise was found. This is due to the surrounding beam pipe regions without clearing electrode, where an electron cloud can still persist.

\section{F. Buildup time of the electron cloud}

Without magnetic field or clearing voltage, the buildup of the EC is exponential, until the curve bends and tends towards a saturation value. In Fig. 10 the signal of pickup \#2 is plotted for the second last turn of the LHC beam. The raw EC signal was averaged over $25 \mathrm{~ns}$ time windows (one value per bunch) and normalized to the maximum value that is reached at the end of the bunch train. Finally, for an 

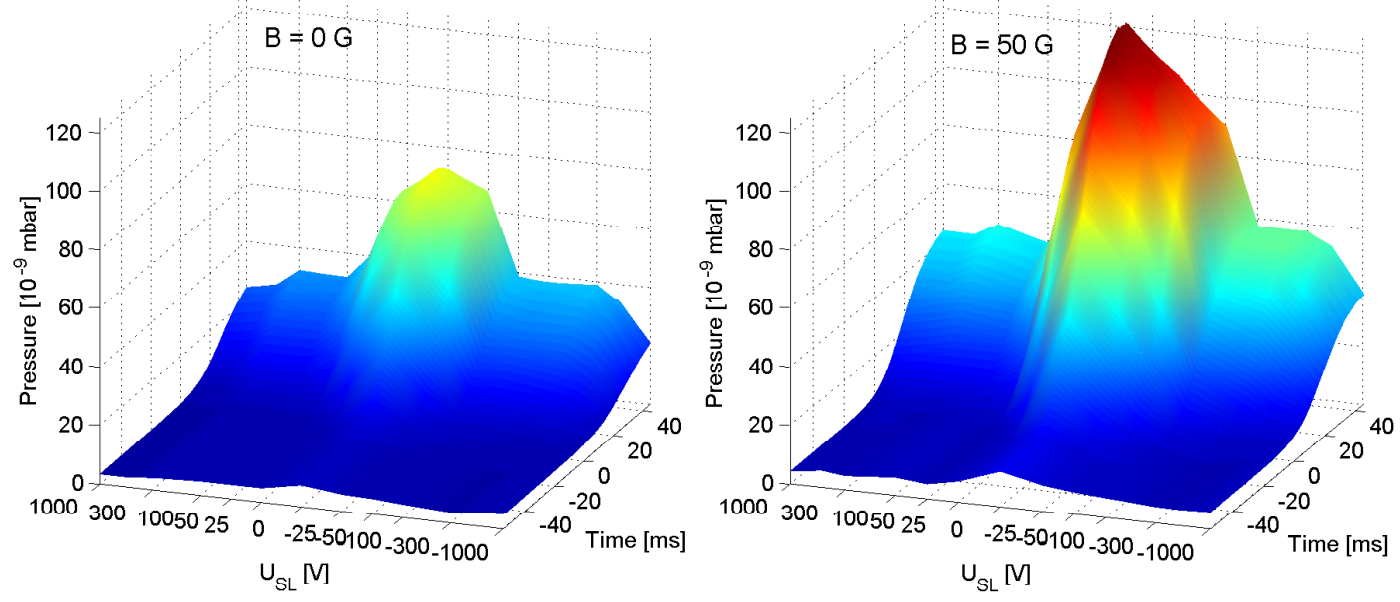

VIDEO 2. Vacuum pressure rise as a function of the stripline clearing voltage $U_{\mathrm{SL}}$ and time in the PS cycle. Even for large clearing voltages a pressure rise can be observed, though the $\Delta p$ is delayed, as the pressure bump originates from the vacuum chamber outside the clearing electrode. With magnetic field the pressure rise is stronger and starts earlier. This video shows the time evolution of the vacuum pressure for $B=0$.

easy comparison with an exponential, the curves were inverted. Even with small clearing voltages the curves generally closely follow exponentials, but the time constants increase for larger clearing voltages.

The buildup time is strongly dependent on the bunch length, since in most cases it considerably decreases during the PS cycle, when the proton bunches are shortened. Without magnetic field in general there is a clear negative correlation between the buildup time and the maximum EC signal (see Fig. 11). In order to include also cases with nonexponential buildup, the $50 \%$ EC rise time was used as a more generally applicable buildup time. In general, with magnetic field and without clearing voltage the buildup

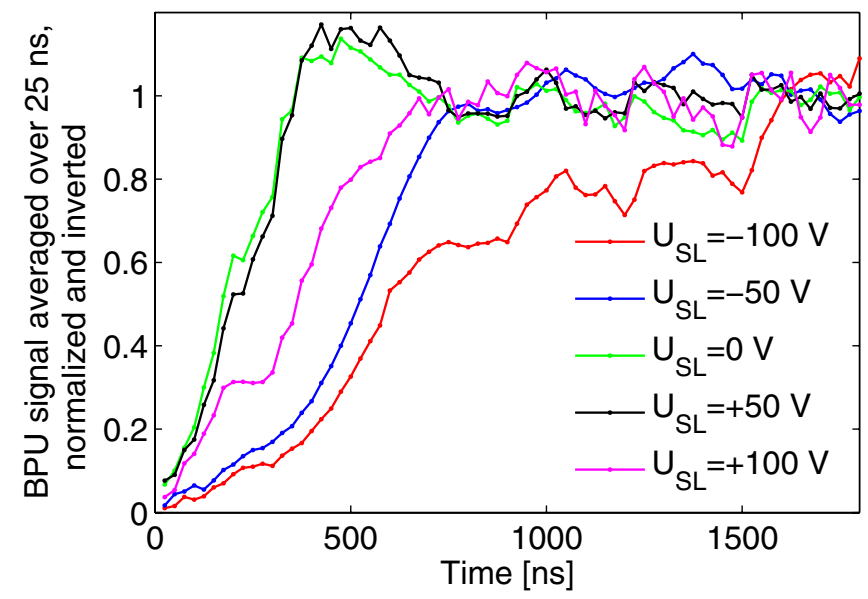

FIG. 10. (Color) Electron cloud buildup time for small clearing voltages without magnetic field. The raw signal from the pickup \#2 was averaged for each bunch, normalized to the maximum signal value in the bunch train and inverted. Without magnetic field the curves closely follow exponentials. time decreases, as well as for larger intensities. However, for certain combinations of magnetic field and clearing voltage $\left(B \geq 25 \mathrm{G}, \quad-100 \mathrm{~V} \leq U_{\mathrm{SL}} \leq-500 \mathrm{~V}\right)$, the buildup departed strongly from exponentials. In such cases, the EC saturation value may be higher than without electric and magnetic field, but it takes much longer to reach this value. This phenomenon is illustrated in Fig. 12, where the buildup time for the second last turn is color coded. For negative clearing voltages with magnetic field, the buildup time can exceed $1 \mu \mathrm{s}$. However, comparing with the last plot in Video 1 shows that in spite of the slow buildup very high saturation values are reached.

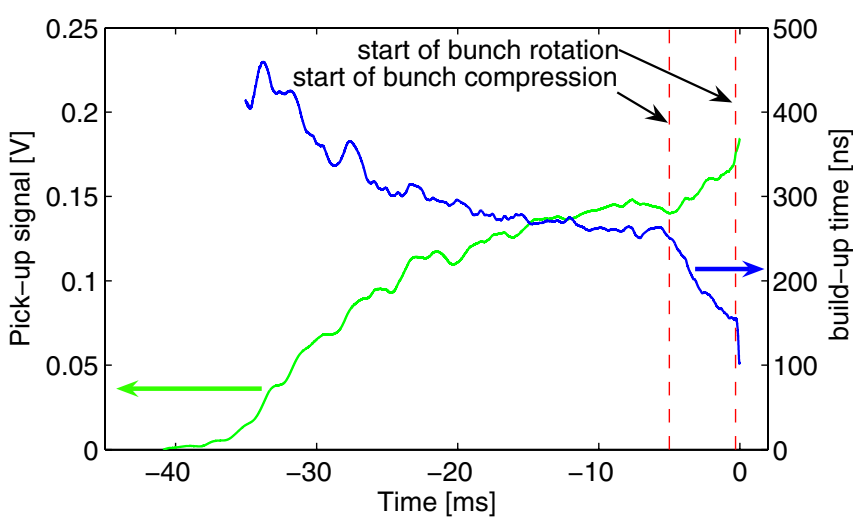

FIG. 11. (Color) Electron cloud buildup time (50\% rise time) compared to the maximum electron signal at the end of the bunch train. As the bunch length decreases during the last tens of milliseconds before ejection, the EC signal increases while its buildup is accelerated. The bunch compression and rotation have a very distinct effect on both EC parameters. 


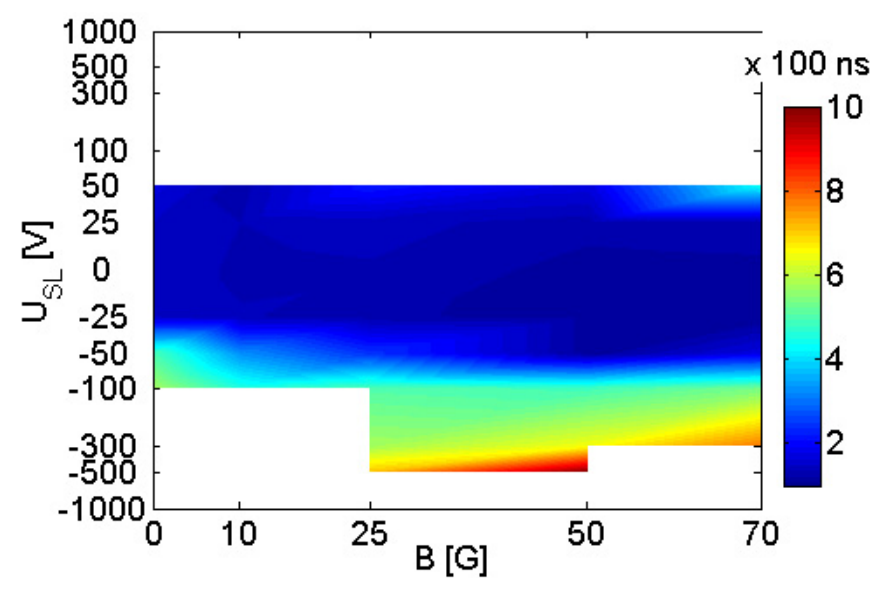

FIG. 12. (Color) Electron cloud buildup time measured with pickup \#2 for the second last turn. The positions in the parameter space where the EC did not reach saturation are left blank in the plot. With magnetic field the buildup time shortens a bit, while it increases appreciably for negative clearing voltages.

\section{G. Decay time of the electron cloud}

After the passage of the last bunch in the bunch train, the EC signal on the pickups returns back to the baseline [see Fig. 5 (right)]. If the electrons get lost at a constant rate, the electron cloud "decay" should follow an exponential curve. Such behavior was found without clearing voltage. However, for negative clearing voltages a second dip was found after the last bunch, which is caused by secondary electrons being liberated from the stripline electrode.

The magnetic field has a significant effect on the EC decay, as well, as it alters the electron trajectories, keeping them in the center of the beam pipe. Figure 13 shows the time constant $\tau$ of the exponential decay that was extracted from the raw data. For a magnetic field of $50 \mathrm{G}, \tau$ is almost doubled compared with the field-free case. However, $\tau$

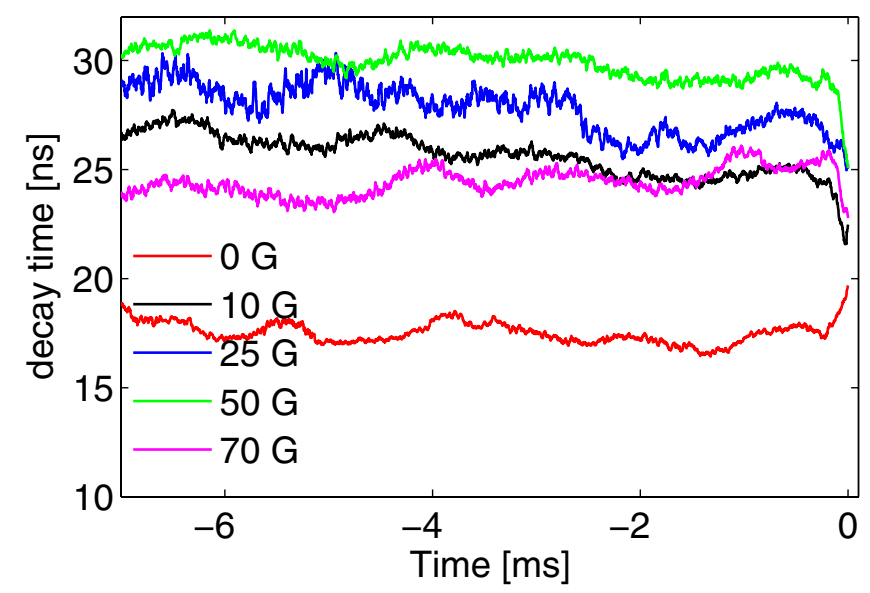

FIG. 13. (Color) Electron cloud decay time $\tau$ during the last $7 \mathrm{~ms}$ of the PS cycle for different magnetic fields. The decay time is not very sensitive on the bunch length, but increases when a magnetic field is present. does not rise monotonically with the magnetic field. During the last $7 \mathrm{~ms}$ before ejection $\tau$ does not vary much, except during the bunch rotation (last $300 \mu \mathrm{s}$ ). This indicates that the bunch length does not have a very strong impact on the EC decay, which is plausible since the bunch length can influence the EC decay only in an indirect way, e.g., by changing the electron energy distribution.

\section{H. Clearing current}

In addition to the vacuum pressure and the pickup signals, the current $I_{\mathrm{SL}}$ on the clearing electrode was measured. Experiments were only performed for large enough clearing voltages such that the EC is weak and the stripline does not draw too much current. The results for both polarities of $U_{\mathrm{SL}}$ are depicted in Fig. 14. For $U_{\mathrm{SL}}>0, I_{\mathrm{SL}}$ is rather large and essentially independent of $U_{\mathrm{SL}}$. In this mode of operation, the clearing electrode basically works by collecting most of the primary electrons. On the other hand, when $U_{\mathrm{SL}}$ becomes more and more negative, $I_{\mathrm{SL}}$ keeps on dropping. For $U_{\mathrm{SL}}=$ $-100 \mathrm{~V}$ a current $I_{\mathrm{SL}}=1 \mu \mathrm{A}$ was measured. A clearing electrode with a highly resistive coating could be used in such a regime. With small magnetic fields $(B=10 \mathrm{G})$ similar stripline currents were found.

In all cases the stripline electrode had the tendency to discharge. For $U_{\mathrm{SL}}>0$ this is due to electron capture, while for $U_{\mathrm{SL}}<0$ it charges positively due to secondary emission induced by high-energy electrons which still reach the electrode. When the stripline electrode was connected to ground over a $1 \mathrm{M} \Omega$ resistor, it tended to charge up positively with a maximum current of $\approx 2 \mu \mathrm{A}$ (or $\approx 5 \mu \mathrm{A}$ for $B=10 \mathrm{G}$ ). This observation suggests that the SEY of the electrode, averaged over the energy distribution of the impinging electrons, is larger than unity.

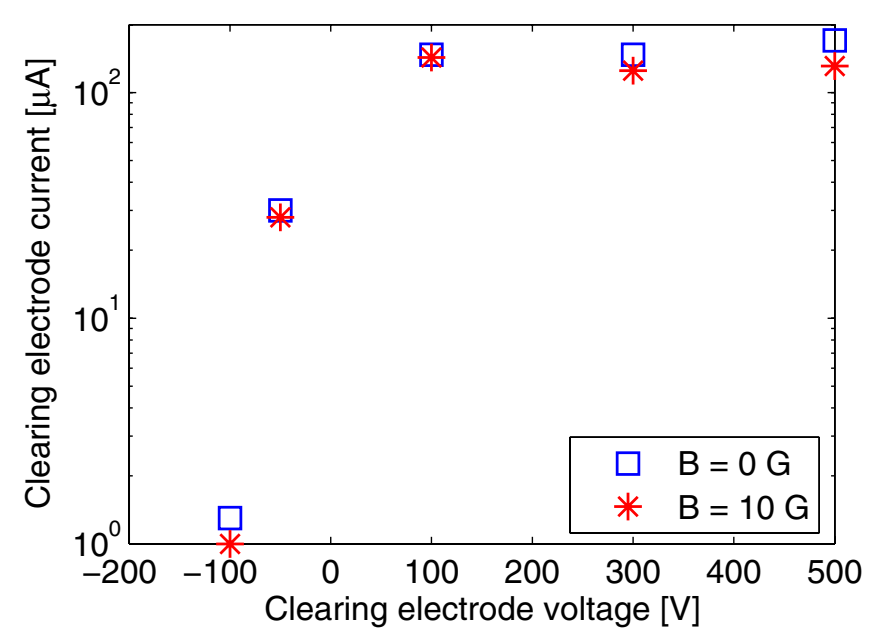

FIG. 14. (Color) The current drawn by the $40 \mathrm{~cm}$ long stripline electrode strongly depends on the polarity of the clearing voltage. For positive $U_{\mathrm{SL}}$ the current is rather large and essentially constant, while it is small for negative $U_{\mathrm{SL}}$. 


\section{CONCLUSION AND OUTLOOK}

A dedicated electron cloud experiment was designed, built, and installed in straight section 98 of the CERN Proton Synchrotron. The equipment comprises a shielded Penning gauge for fast vacuum logging, two shielded button pickups, a dipole magnet, as well as a stripline electrode for electron cloud clearing. Starting from June 2007 clear signs of an electron cloud were found in the PS with nominal LHC beam. Several indications strongly point to an electron cloud effect: a fast vacuum pressure rise, a characteristic signal on the shielded button pickups, as well as a current on the clearing electrode. The effect develops during the last 40 to $50 \mathrm{~ms}$ before ejection, when the bunches are shortened by the rf gymnastics. The electron cloud can be suppressed by putting a sufficiently large voltage of either polarity onto the clearing electrode. A parameter space spanned by magnetic fields up to $70 \mathrm{G}$ and clearing voltages up to $\pm 1 \mathrm{kV}$ was scanned for electron cloud formation. Characteristic islands with surviving electron cloud were found for some clearing voltages and magnetic fields. However, full electron cloud suppression was always obtained for high enough clearing voltages of either sign. Furthermore, the electron cloud buildup at the beginning of a bunch train and its decay after the passage of the last bunch were studied for different magnetic fields and clearing voltages.

One of the important findings discussed here is the fact that electron cloud suppression is possible for both positive and negative bias of the clearing electrode. For the nominal LHC beam in the PS and the used electrode geometry, the clearing current was in the order of $1 \mu \mathrm{A}$ for a negative bias voltage and typically 2 orders of magnitude larger for a positive bias voltage. The low current drawn for negative bias opens up the possibility for "invisible" clearing electrodes with very low beam coupling impedance [20]. Such structures, which consist of a highly resistive coating $\left(\mathrm{R}_{\text {surface }} \gg 377 \Omega\right)$ on a dielectric substrate could be implemented all along an accelerator beam pipe by using, e.g., enamel or a thin glass layer technology.

\section{ACKNOWLEDGMENTS}

We would like to thank especially the CERN Main Workshop for their fast reaction and effort in fabricating and cleaning all new vacuum equipment within very short notice. Thanks to Nicholas Sammut for magnetic field measurements on the dipole magnet and Eric Page for the fast vacuum logging system. Special thanks to Steven Hancock for beam parameter measurements and his patient support and tutorials on operational aspects, and to Gianluigi Arduini, Heiko Damerau, Jose Miguel Jimenez, Rende Steerenberg, and Frank Zimmermann for inspiring discussions. The support from Elena Shaposhnikova, Trevor Linnecar, and Pierre Strubin is highly acknowledged.
[1] G. I. Budker, G. I. Dimov, and V.G. Dudnikov, in Proceedings of the International Symposium on Electron and Positron Storage Rings, Saclay, 1966 (Universitaiers De France, Orsay, 1966), p. VIII-6-1.

[2] O. Gröbner, in Proceedings of the 10th International Conference on High Energy Accelerators, Protvino, Russia, 1977 (Institute of High Energy Physics, Protvino, 1977).

[3] A. Kulikov, A. Fisher, S. Heifets, J. Seeman, M. Sullivan, U. Wienands, and W. Kozanecki, in Proceedings of the Particle Accelerator Conference, Chicago, IL, 2001 (IEEE, New York, 2001), p. 1903.

[4] G. Arduini et al., in Proceedings of the Seventh European Particle Accelerator Conference, Vienna, 2000, edited by J. Poole and Ch. Petit-Jean-Genaz (European Physical Society, Geneva, 2000), p. 259.

[5] H. Fukuma et al., in Proceedings of the Seventh European Particle Accelerator Conference, Vienna, 2000 (Ref. [4]), p. 1122.

[6] R. Cappi, M. Giovannozzi, E. Métral, G. Métral, G. Rumolo, and F. Zimmermann, Phys. Rev. ST Accel. Beams 5, 094401 (2002).

[7] G. Arduini, in Proceedings of the 3rd CARE-HHH-APD Workshop: Towards a Roadmap for the Upgrade of the LHC and GSI Accelerator Complex-LHC-LUMI-06, 2006, Valencia, Spain, edited by W. Scandale, T. Taylor, and F. Zimmermann, CERN Yellow Report No. CERN2007-002/CARE-Conf-07-004-HHH/CARE-Conf-2007004-HHH, p. 159.

[8] G. Arduini, J. M. Jimenez, and K. Weiss, in Proceedings of the 2001 Particle Accelerator Conference, Chicago, 2001, (Ref. [3]), p. 685.

[9] A. Rossi, in Proceedings of the 31st ICFA Beam Dynamics Workshop on Electron-Cloud Effects ECLOUD'04, Napa, CA, 2004, edited by M. Furman, S. Henderson, and F. Zimmermann, CERN Yellow Report No. CERN-2005-001/CARE-Conf-05-001-HHH/LBNL 56372/SNS-10400000-TR0024-R00, p. 113.

[10] F. Le Pimpec, R. E. Kirby, F. K. King, and M. Pivi, Nucl. Instrum. Methods Phys. Res., Sect. A 564, 44 (2006).

[11] L. Wang, T. O. Raubenheimer, and G. Stupakov, Nucl. Instrum. Methods Phys. Res., Sect. A 571, 588 (2007).

[12] M. Venturini, in Proceedings of the Joint CARE-ELAN, CARE-HHH-APD, and EUROTEV-WP3 Workshop on Electron Cloud Clearing-Electron Cloud Effects and Technological Consequences ECL2, CERN, Geneva, Switzerland, 2007, edited by F. Caspers, W. Scandale, D. Schulte, and F. Zimmermann, CERN Report No. CERNAB-2007-064-ABP/CARE-ELAN-Document-2007-006/ EUROTEV-Report-2007-060/CARE-Conf-07-007-HHH/ CARE-Conf-2007-007-HHH, p. 6.

[13] L. Wang, A. Chao, H. Fukuma, S. Kurokawa, and S. S. Win, in Proceedings of the 31st ICFA Beam Dynamics Workshop on Electron-Cloud Effects ECLOUD'04, Napa, CA, 2004, Ref. [9], p. 255.

[14] L. F. Wang, D. Raparia, J. Wei, and S. Y. Zhang, Phys. Rev. ST Accel. Beams 7, 034401 (2004).

[15] F. J. Decker, F. Caspers, and F. Zimmermann, in Proceedings of the Mini Workshop on Electron Cloud Simulations for Proton and Positron Beams- 
ECLOUD'02, CERN, Geneva, Switzerland, 2002, edited by G. Rumolo and F. Zimmermann, CERN Yellow Report No. CERN-2002-001, p. 87.

[16] F. Pedersen, A. Poncet, and L. Søby, in Proceedings of the 1989 Particle Accelerator Conference, Chicago, IL (IEEE, Piscataway, NJ, 1989), Vol. 3, p. 1786.

[17] J. Wei, M. Blaskiewicz, W. Fischer, H. C. Hseuh, U. Iriso, T. Roser, L. Wang, and S. Y. Zhang, in Proceedings of the 1st CARE-HHH-APD Workshop on Beam Dynamics in Future Hadron Colliders and Rapidly Cycling HighIntensity Synchrotrons-HHH 2004, CERN, Geneva, Switzerland, 2004, edited by F. Ruggiero, W. Scandale, and F. Zimmermann, CERN Report No. CERN-2005-006 /CARE-Conf-05-002-HHH/CARE-Conf-2005-002-HHH, p. 43.

[18] P.F. Tavares, in Proceedings of the 1992 European Particle Accelerator Conference, Berlin, Germany, 1992, edited by H. Henke, H. Homeyer, and C. Petit-Jean-Genaz (Editions Frontieres, Gif-sur-Yvette, France, 1992), p. 1647.
[19] B. Spataro and M. Zobov, Daథne Report No. G-64, Frascati, 2005 (unpublished).

[20] T. Kroyer, F. Caspers, E. Metral, and F. Zimmermann, in Proceedings of the Joint CARE-ELAN, CARE-HHHAPD, and EUROTEV-WP3 Workshop on Electron Cloud Clearing-Electron Cloud Effects and Technological Consequences ECL2, CERN, Geneva, Switzerland, 2007, in Ref. [20], p. 64.

[21] F. Zimmermann, CERN Report No. CERN-SL-Note2001-022 (AP), Geneva, 2001 (unpublished).

[22] W. Bruns, in Proceedings of the Joint CARE-ELAN, CARE-HHH-APD, and EUROTEV-WP3 Workshop on Electron Cloud Clearing-Electron Cloud Effects and Technological Consequences ECL2, CERN, Geneva, Switzerland, 2007, in Ref. [20], p. 30.

[23] T. Kroyer and N. Sammut, CERN Report No. EDMS 829360, 2007 (unpublished).

[24] LHC Design Report, edited by M. Benedikt, P. Collier, V. Mertens, J. Poole, and K. Schindl, Geneva, 2004, Vol. III, p. 45. 